\title{
Large Scale, Long-Term, High Granularity Measurement of Active Travel Using Smartphones Apps ${ }^{\dagger}$
}

\section{Ben W. Heller ${ }^{1, *}$, Suvodeep Mazumdar ${ }^{2}$ and Fabio Ciravegna ${ }^{3}$}

1 Centre for Sports Engineering Research, Faculty of Health and Wellbeing, Sheffield Hallam University, 11 Broomgrove Road, Sheffield S10 2LX, UK

2 Department of Computing, Sheffield Hallam University, City Campus, Howard Street, Sheffield S1 1WB, UK; S.Mazumdar@shu.ac.uk

3 Department of Computer Science, University of Sheffield, 211 Portobello, Sheffield S10 2TN, UK; f.ciravegna@sheffield.ac.uk

* Correspondence: b.heller@shu.ac.uk; Tel.: +44-114-225-5555

+ Presented at the 12th Conference of the International Sports Engineering Association, Brisbane, Queensland, Australia, 26-29 March 2018.

Published: 24 February 2018

\begin{abstract}
Accurate, long-term data are needed in order to determine trends in active travel, to examine the effectiveness of any interventions and to quantify the health, social and economic consequences of active travel. However, most studies of individual travel behaviour have either used self-report (which is limited in detail and open to bias), or provided logging devices for short periods, so lack the ability to monitor long-term trends. We have developed apps using participants' own smartphones (Android or iOS) that monitor and feed-back individual user's physical activity whilst the phone is carried or worn. The nature, time and location of any physical activity are uploaded to a secure survey and allow researchers to identify large scale behaviour. Pilot data from almost 2000 users have been logged and are reported. This constitutes a natural experiment, collecting long-term physical activity, transport mode and route choice information across a large cross-section of users.
\end{abstract}

Keywords: mobile devices; active travel; physical activity

\section{Introduction}

Active travel (walking running or cycling for commuting or other utilitarian purposes) has individual health benefits and is one of the most acceptable ways to meet government recommended levels of physical activity (PA), which is associated with a 30\% risk reduction in all-cause mortality [1]. Studies suggest that obesity is positively associated with motorized vehicle travel or negatively associated with active travel (or both) across a broad cross-section of nations [2]. A recently released study of 264,277 UK participants with median 5 year follow-up [3] has shown that cycling to work is linked to a substantial decrease in the risk of developing and dying from cancer or heart disease and all-cause mortality. People who walked to work, covering more than six miles a week had a lower risk of CVD incidence; they also had a lower risk of CVD mortality, but walking had no statistically significant impact on all-cause mortality or cancer outcomes. A study in Barcelona of the Bicing public bicycle sharing initiative found an annual increase in mortality for Barcelona residents of 0.03 deaths from road traffic accidents and 0.13 deaths from air pollution, but a reduction of 12.46 deaths annually due to increased physical activity, giving a benefit ratio of 77 [4].

An editorial in the British Medical Journal referring to the Celis-Morales study states: 
The findings from this study are a clear call for political action on active commuting, which has the potential to improve public health by preventing common (and costly) non-communicable diseases. A shift from car to more active modes of travel will also decrease traffic in congested city centres and help reduce air pollution, with further benefits for health [5].

Accurate, long-term data are needed in order to accurately determine the health and economic consequences of active travel, and to examine the effectiveness of any interventions. However, Saunders et al. [6] identify limitations of studies of transportation choice to date:

1. Lacking long-term data describing physical activity and travel behaviours.

2. Having limited generalizability due to geographically limited scope.

3. Being open to potential bias by relying on self-reported data.

Recently developed measurement devices combining GPS, global information systems (GIS) and inertial measurement unit (IMU) sensors allow researchers to capture objectively measured data about a participant's location, surrounding environment, and physical movements. However, these sensors are limited with practical issues including limited battery life and unreliable satellite signals [7]. Modern smartphones have effectively solved these problems by combining long-battery-life, IMU sensors and redundancy of location detection, allowing large-scale, long-term, geographically distributed studies, with the additional benefit of near-real-time data upload.

\section{Methods}

\subsection{System}

The system philosophy was to minimise disruption to an individual's activities, so activity was detected in the background with no requirement for users to start and stop recording. Other requirements were to accurately detect location during activities, and to minimise battery drain. These two requirements were contradictory so a relatively low frequency of location recording was used of one location request every $45 \mathrm{~s}$. Apps were developed for iOS and Android devices, using the operating systems' built-in movement detection algorithms to identify periods of activity and then log these together with time and location. Activity data were compiled and transmitted to a central server every few hours to minimise data and battery usage.

The app was titled the MoveMore App and was originally released as part of a programme (Move More Month) to encourage the people of Sheffield to be more active immediately prior to the Rio Olympic Games in July 2016. Total minutes of activity (walking, cycling and running) were displayed by the app for each individual, together with a graphic representing a daily medal. These were determined as

$\begin{array}{ll}\text { - } & \text { Bronze } 30 \mathrm{~min} \\ \text { - } & \text { Silver } 45 \mathrm{~min} \\ \text { - } & \text { Gold } 60 \mathrm{~min} \\ \text { Black } 90 \mathrm{~min}\end{array}$

Example screen shots are shown in Figure 1.

\subsection{Participants}

Following institutional ethical approval, participants were recruited via participating employers, marketing initiatives at local events, local and national media and word of mouth. Guidance for download emphasised that the app was not to be used by anyone aged under 18 . Participants entered their gender and age when registering for the app. 

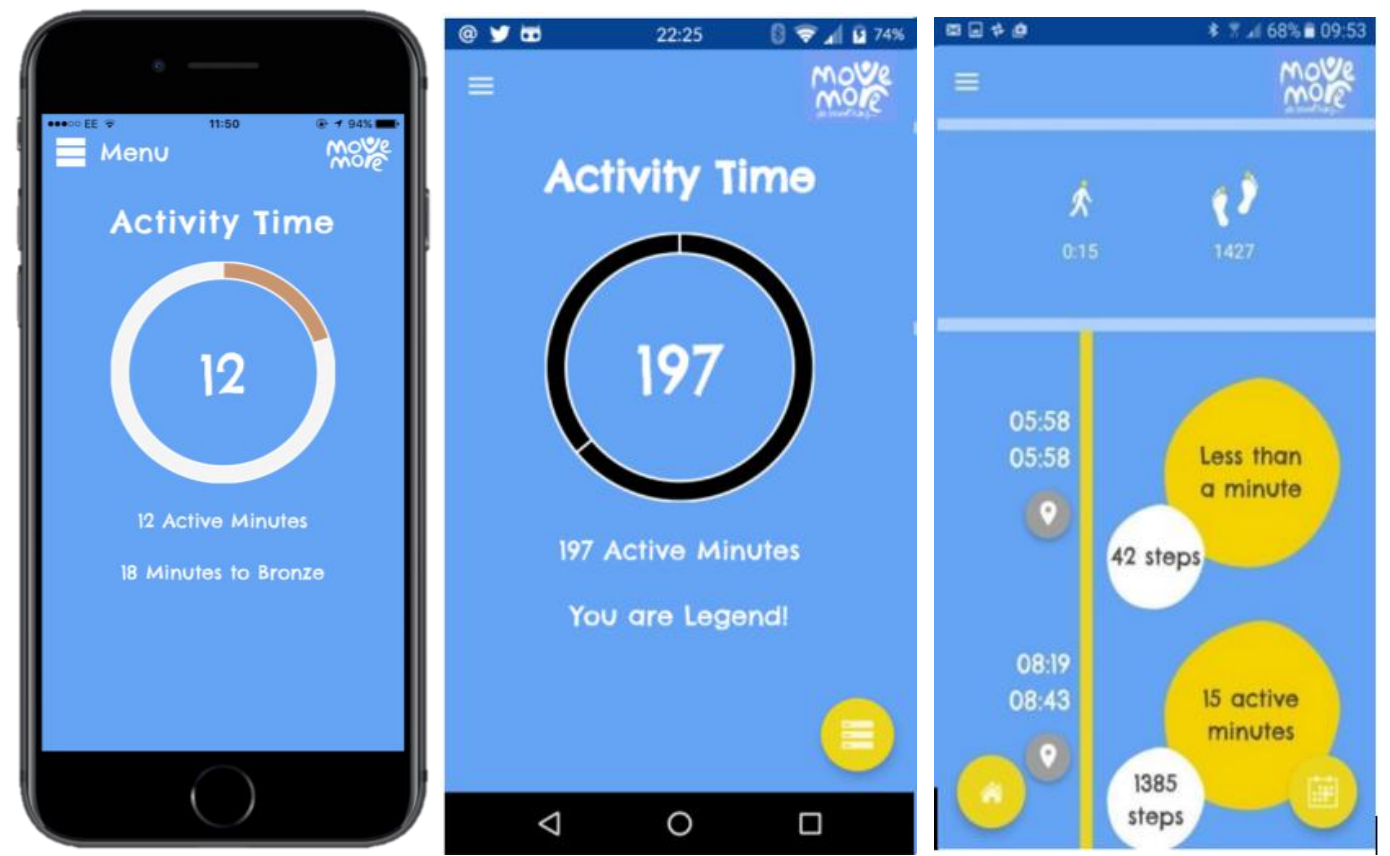

Figure 1. MoveMore app screenshots.

\subsection{Data Storage}

Activity and location data were uploaded to a secure server at the University of Sheffield. These data were only identifiable through a reference code, but as they contained location information that revealed home and work locations and also patterns of behaviour, access was restricted to only immediate members of the project team.

\subsection{Data Analyses}

The data were analysed according to time, location and activity type. Raw location coordinates were fed through the Open Street Map routing engines (cycling or walking as appropriate) to identify individual road usage

\section{Results}

In total 1972 users downloaded the app during the month, of which 1282 were on iOS and 690 on Android. The participants were 37\% male and 63\% female. Their age breakdown can be seen in Figure 2. The distribution of users against postcode within Sheffield is shown in Figure 3.

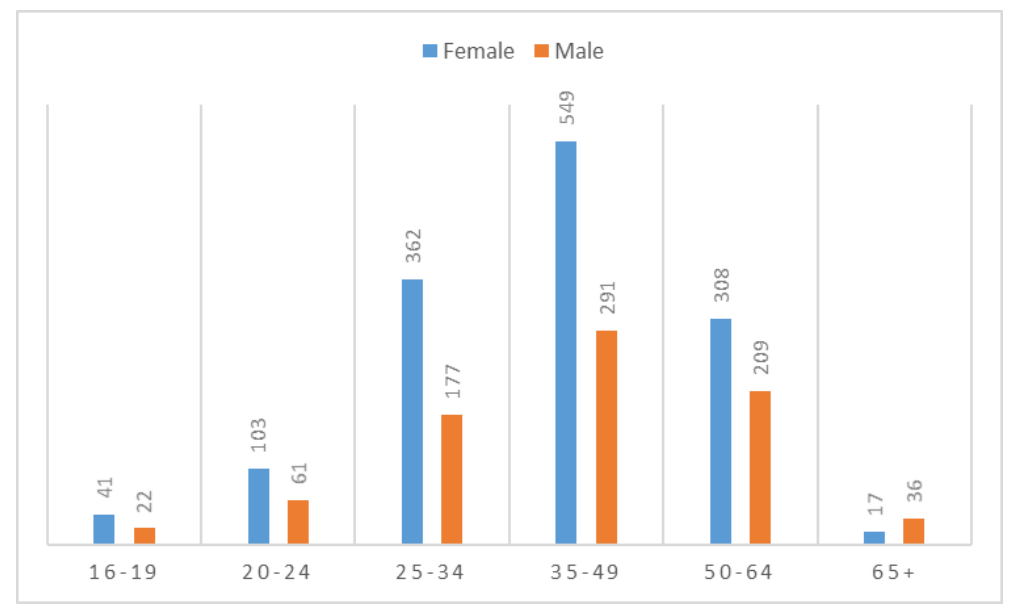

Figure 2. Breakdown of gender and age. The total sum of users (2176) is higher than that given previously as these results were obtained after the "Move More Month" finished. 


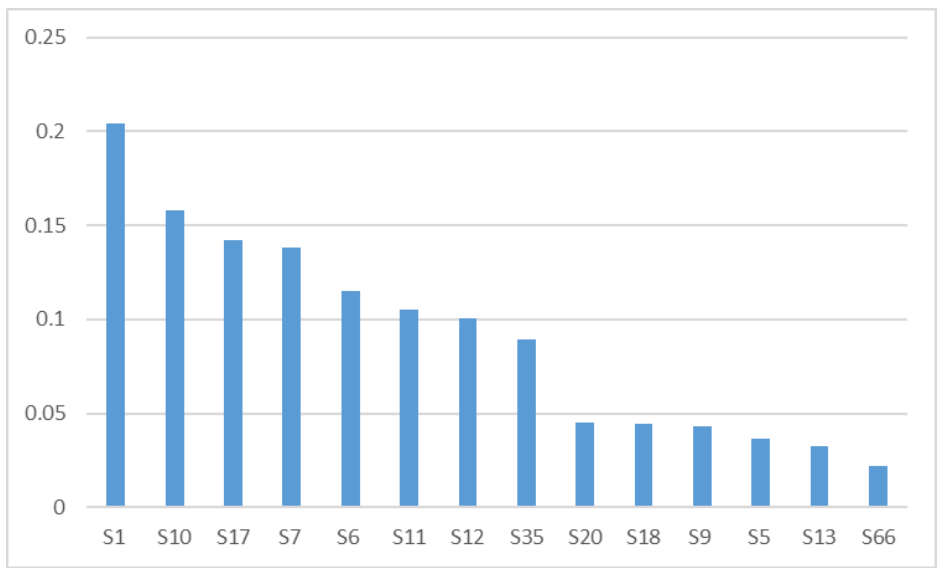

Figure 3. Percentage of app users by Sheffield postcodes.

Distribution of all activity against time of day is shown in Figure 4, with a distribution of running activity alone against time in Figure 5.

Geographical distribution of all activity over the month is shown in Figure 6.

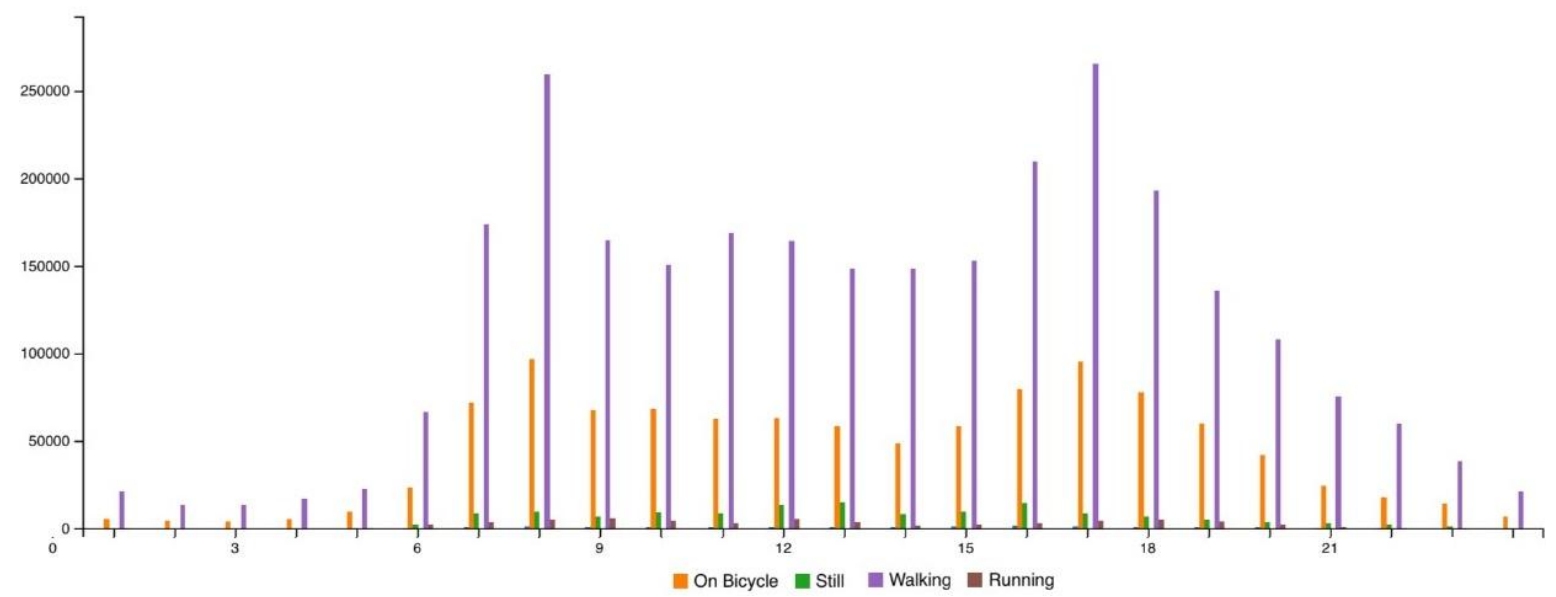

Figure 4. Distribution of minutes of all activities against time of day for all days.

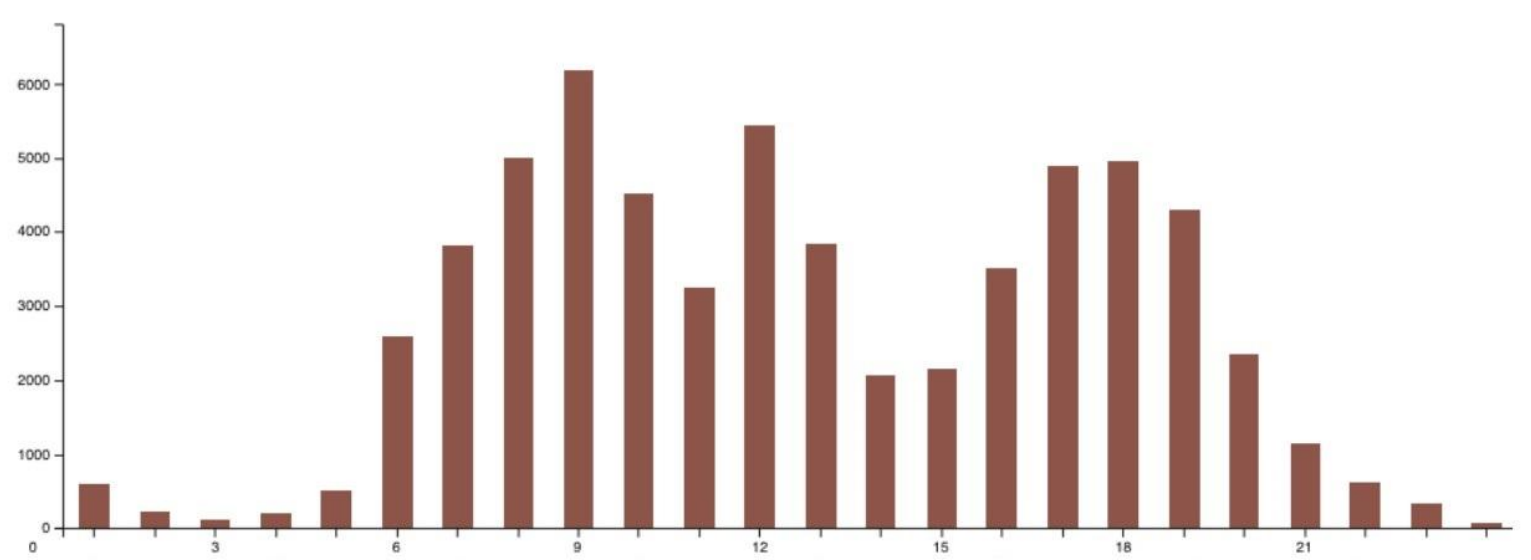

Figure 5. Distribution of minutes of running against time of day for all days. 


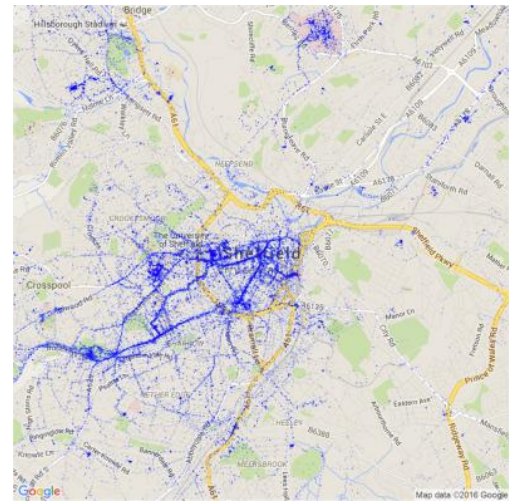

(a)

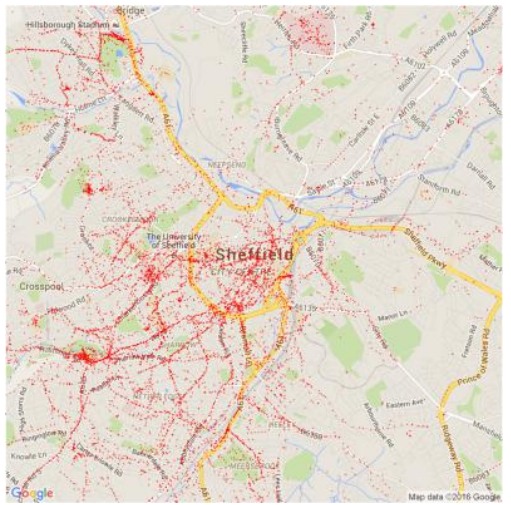

(b)

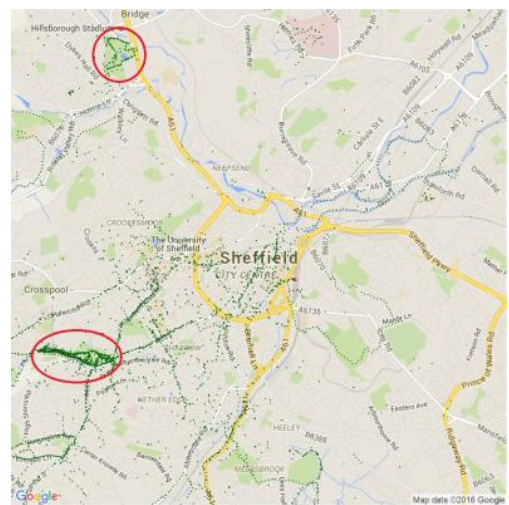

(c)

Figure 6. Geographical distribution of (a) walking; (b) cycling; (c) running. parkruns highlighted.

\section{Discussion}

This month-long data collection showed the possibility of using participants' own smartphones to collect detailed data on their physical activity. Two-thousand users represents approximately $0.4 \%$ of the population of Sheffield. It is clear from the app distribution against postcode that there is a bias towards certain areas; these tend to be Sheffield's more prosperous areas with white collar-workers or with high student populations.

The domination of walking and cycling over running shown in Figure 4. Distribution of minutes of all activities against time of day for all days Figure 4, together with the bimodal distribution around commuting times suggest that active travel constitutes a significant part of this population's weekly physical activity. An examination of running alone in Figure 5 shows a similar pattern of peaks before and after typical work hours, with a morning peak later at 9 am (probably influenced by the popularity of parkrun, held at 9 am on Saturdays in the UK). Compared to patterns for cycling and walking, an additional peak at midday is evident, suggestive of lunch-time (recreational) running.

Figure 6 shows a dominance of activity in the south-west of the city. Whilst this represents one of the more attractive areas of Sheffield (with access to the National Park), it also reflects the distribution of app users in these student/professional areas. The routes of 2 parkruns can be seen in the running figure-demonstrating their popularity with this population.

The results shown here are just a small example of the analyses that can be performed on this rich, large dataset. Other analyses include monitoring specific roads or public areas to observe patterns of use over time, and the effect of weather and sunrise/sunset times on behaviour. The effect of an intervention e.g., (provision of improved cycling infrastructure) can potentially be observed, given sufficient users.

The advantages of the technique include:

- Very high scalability: collection can be easily made from thousands of participants with little additional effort.

- Low cost: once the apps are written the additional cost per user is effectively zero.

- High reliability: users are already motivated to charge their phones and carry them with them.

- Low reactivity: activity detection is automatic and users do not need to interact with the app.

Disadvantages include

- Smartphones are not carried for all activities (e.g., swimming), and so will underestimate activity.

- Not everyone has access to a smartphone, or the digital literacy to be able to use one. This may disproportionally apply to older people.

- There is a cost to the user in battery life and memory. Whilst we have attempted to minimise this, some users will consequently uninstall the app.

- The data are sensitive and must be carefully secured against unauthorised access.

- The app didn't work well on all phones, particular iPhones prior to $5 \mathrm{~s}$ and older Android OS versions. This often led to users uninstalling the app. 
The software developed within the MoveMore App has been further developed into the Active10 app funded by Public Health England. Through extensive marketing this app currently (September 2017) has over 300,000 users, offering a fantastically rich resource to study population-scale physical activity.

\section{Conclusions}

An app to automatically detect type, time and location of physical activity has been developed, it shows great potential in studying physical activity and active travel.

Acknowledgments: This research was in-part funded by the Horizon 2020 science programme of the European Union, under the SETA project funded under the H2020-ICT-2015 scheme.

Conflicts of Interest: The authors declare no conflict of interest.

\section{References}

1. Department of Health. Start Active, Stay Active: A Report on Physical Activity for Health from the Four Home Countries Chief Medical Officers; Department of Health: London, UK, 2011.

2. King, D.M.; Jacobson, S.H. What is driving obesity? A review on the connections between obesity and motorized transportation. Curr. Obes. Rep. 2017, 6, 3-9.

3. Celis-Morales, C.A.; Lyall, D.M.; Welsh, P.; Anderson, J.; Steell, L.; Guo, Y.; Maldonado, R.; Mackay, D.F.; Pell, J.P.; Sattar, N.; et al. Association between active commuting and incident cardiovascular disease, cancer, and mortality: Prospective cohort study. BMJ 2017, 357, j1456.

4. Rojas-Rueda, D.; de Nazelle, A.; Tainio, M.; Nieuwenhuijsen, M.J. The health risks and benefits of cycling in urban environments compared with car use: Health impact assessment study. BMJ 2011, 343, d4521.

5. Andersen, L.B. Active commuting is beneficial for health. BMJ 2017, 357, j1740.

6. Saunders, L.E.; Green, J.M.; Petticrew, M.P.; Steinbach, R.; Roberts, H. What are the health benefits of active travel? A systematic review of trials and cohort studies. PLoS ONE 2013, 8, e69912.

7. Oliver, M.; Badland, H.; Mavoa, S.; Duncan, M.J.; Duncan, S. Combining GPS, GIS, and accelerometry: Methodological issues in the assessment of location and intensity of travel behaviors. J. Phys. Act. Health 2010, 7, 102-108.

(C) 2018 by the authors. Licensee MDPI, Basel, Switzerland. This article is an open access article distributed under the terms and conditions of the Creative Commons Attribution (CC BY) license (http://creativecommons.org/licenses/by/4.0/). 